\title{
Assets of imputation to ultra-high density for productive and functional traits
}

\author{
J. A. Jiménez-Montero, ${ }^{* 1}$ D. Gianola, $¥ \S$ K. Weigel,† R. Alenda, ${ }^{*}$ and O. González-Recio\#II \\ *Departamento de Producción Animal E.T.S.I. Agrónomos - Universidad Politécnica de Madrid, Ciudad Universitaria s/n 28040 Madrid, Spain \\ †Department of Dairy Science, \\ ‡Department of Biostatistics and Medical Informatics, and \\ $\S$ Department of Animal Sciences, University of Wisconsin, Madison 53706 \\ \#Departamento de Mejora Genética Animal, Instituto Nacional de Investigación y Tecnología Agraria y Alimentaria (INIA), Crta. La Coruña km 7.5, \\ 28040 Madrid, Spain \\ IIBiosciences Research Division, Department of Environment and Primary Industries Victoria, Bundoora VIC 3083, Australia
}

\begin{abstract}
The aim of this study was to evaluate differentdensity genotyping panels for genotype imputation and genomic prediction. Genotypes from customized Golden Gate Bovine3K BeadChip [LD3K; low-density (LD) 3,000-marker (3K); Illumina Inc., San Diego, CA] and BovineLD BeadChip [LD6K; 6,000-marker (6K); Illumina Inc.] panels were imputed to the BovineSNP50v2 BeadChip [50K; 50,000-marker; Illumina Inc.]. In addition, LD3K, LD6K, and 50K genotypes were imputed to a BovineHD BeadChip [HD; high-density 800,000-marker (800K) panel], and with predictive ability evaluated and compared subsequently. Comparisons of prediction accuracy were carried out using Random boosting and genomic BLUP. Four traits under selection in the Spanish Holstein population were used: milk yield, fat percentage (FP), somatic cell count, and days open (DO). Training sets at $50 \mathrm{~K}$ density for imputation and prediction included 1,632 genotypes. Testing sets for imputation from LD to $50 \mathrm{~K}$ contained 834 genotypes and testing sets for genomic evaluation included 383 bulls. The reference population genotyped at HD included 192 bulls. Imputation using BEAGLE software (http://faculty.washington.edu/browning/beagle/beagle.html) was effective for reconstruction of dense $50 \mathrm{~K}$ and HD genotypes, even when a small reference population was used, with $98.3 \%$ of SNP correctly imputed. Random boosting outperformed genomic BLUP in terms of prediction reliability, mean squared error, and selection effectiveness of top animals in the case of FP. For other traits, however, no clear differences existed between methods. No differences were found between imputed LD and 50K genotypes, whereas evaluation of genotypes imputed to HD was on average across data set, method, and trait, $4 \%$ more accurate than $50 \mathrm{~K}$ prediction, and showed smaller $(2 \%)$ mean squared error of
\end{abstract}

Received March 12, 2013.

Accepted May 27, 2013.

${ }^{1}$ Corresponding author: joseantonio.jimenez.montero@upm.es predictions. Similar bias in regression coefficients was found across data sets but regressions were 0.32 units closer to unity for DO when genotypes were imputed to HD density. Imputation to HD genotypes might produce higher stability in the genomic proofs of young candidates. Regarding selection effectiveness of top animals, more $(2 \%)$ top bulls were classified correctly with imputed LD6K genotypes than with LD3K. When the original $50 \mathrm{~K}$ genotypes were used, correct classification of top bulls increased by $1 \%$, and when those genotypes were imputed to HD, $3 \%$ more top bulls were detected. Selection effectiveness could be slightly enhanced for certain traits such as FP, somatic cell count, or DO when genotypes are imputed to HD. Genetic evaluation units may consider a trait-dependent strategy in terms of method and genotype density for use in the genomeenhanced evaluations.

Key words: imputation, marker density, genomic selection, machine learning

\section{INTRODUCTION}

Genomic selection (GS) in dairy cattle started in 2006 (de Roos et al., 2009), when high-density (HD) SNP panels became affordable for application to livestock and plants (Van Tassell et al., 2008). The first official direct genomic values (DGV) were provided to dairy farmers in 2008 in New Zealand (Harris et al., 2008) and January 2009 in the United States (Wiggans et al., 2009).

Different approaches are currently used for estimating genomic values. Genomic prediction methods can be categorized as (1) methods that regress phenotypic records on SNP markers directly and (2) methods that compute genomic values as a function of the genomic relationship using a (co)variance structure between subjects (de los Campos et al., 2009). The first group of methods includes several Bayesian regression approaches, such as Bayes-A, Bayes-B (Meuwissen et al., 2001), and the Bayesian least absolute shrinkage selection operator (B-LASSO), as described by Park and Casella 
(2008). The second group includes methods that compute a realized relationship matrix from the markers, such as genomic BLUP (G-BLUP; VanRaden et al., 2009), or Single Step (Misztal et al., 2009) methods, to augment or replace the traditional pedigree-based relationship matrix. In addition to the aforementioned approaches, an alternative for dealing with large data sets and complex interactions between SNP is machinelearning algorithms (Long et al., 2007). Boosting algorithms are among the most appealing machine-learning methods for genomic-prediction problems (Ogutu et al., 2011). Efficiency of DGV prediction in dairy cattle can be enhanced by modification to the algorithm, specifically random boosting (R-Boost), as described by González-Recio et al. (2013). Despite the improvement in reliability of young selection candidates achieved with genome-enabled evaluations (Wiggans et al., 2011), the commercial price of HD SNP chips may limit their use to males and elite females in many populations.

A next key objective in GS programs is to optimize the use of genomic information (Pryce and Daetwyler, 2012). Use of low-density (LD) SNP panels with subsequent imputation is a promising form of reducing genotyping costs; this is because use of these LD genotyping panels could greatly increase the number of genotyped animals in commercial dairy herds. The optimal size of such panels depends on population characteristics, such as extent of linkage disequilibrium, genetic architecture of traits under selection, number and proportion of animals with HD SNP genotypes, and genetic relatedness between these animals and future candidates (Meuwissen and Goddard, 2010; Weigel et al., 2010a). Imputation methods work by combining data from a reference panel of individuals genotyped at a dense set of polymorphic sites (usually SNP) with data from a study sample collected from a genetically similar population but genotyped at a subset of these sites (Howie et al., 2009). A need exists to integrate different-density SNP panels in genome-based breeding programs. For accurate imputation of missing SNP, the reference population must include a sufficient number of individuals representative of each SNP allele of the whole population (Hao et al., 2009). Theoretically, heavily represented bulls in the population, or animals from the most common matings (sire $\times$ daughter of a frequently use sire) are optimum animals to be genotyped as a reference population. Moderate-density (50,000-marker, 50K) genotypes can be imputed from LD [2,000- to 4,000-marker (2 to $4 \mathrm{~K})]$ genotypes with accuracy above $90 \%$ (Weigel et al., 2010b).

Currently in cattle, the most commonly used chip is the BovineSNP50.v2 BeadChip (50K; 50,000-marker; Illumina Inc., San Diego, CA) and imputation strategies are focused on imputation from 6,000-marker $(\mathbf{6 K})$ to $50 \mathrm{~K}$. The availability of the $800,000(\mathbf{8 0 0 K})$ SNP BovineHD BeadChip (Illumina Inc.) opens the chance of imputation from 50K to this extra-large HD panel. Genotyping a large reference population at extra-large HD would be cost prohibitive. However, genotyping a subset of this reference population, and then imputing the rest of the genotypes may be an efficient strategy if the predictive ability of subsequent genomic evaluations exceeds that obtained before imputation. In addition, imputed SNP from LD 3,000-marker (3K) and 6K panels to HD must be assessed in terms of predictive ability.

Several methods have been developed for imputation, and software is publicly available for these methods (Scheet and Stephens, 2006; Kong et al., 2008; Howie et al., 2009); BEAGLE (Browning and Browning, 2009) has become one of the preferred options for imputation of large data sets of $3 \mathrm{~K}, 6 \mathrm{~K}$, and $50 \mathrm{~K}$ dairy cattle genotypes (Boichard et al., 2012; Erbe et al., 2012). This software uses a hidden Markov model to infer haplotype phase with both typed and untyped SNP. Their competitive imputation accuracy and computational advantages compared with other methods have been widely reported (Nothnagel et al., 2009; Calus et al., 2011; Segelke et al., 2012; Sun et al., 2012).

After imputation, it is possible to estimate DGV with similar accuracy to that obtained from HD genotyping (Berry and Kearney, 2011). Accuracy of DGV for selection candidates can be increased by imputation compared with estimation based on LD SNP. Recent studies have shown that LD genotypes from animals with enough phenotypic information can be added to the reference population after imputation to increase the overall accuracy of estimation (Weigel et al., 2010a). The objective of this study was to compare imputation accuracy, predictive ability, and selection effectiveness for selection candidates genotyped at different densities using the R-Boost and G-BLUP methods.

\section{MATERIALS AND METHODS}

\section{Genotypes and Phenotypes}

A total of 2,658 genotyped bulls were used in this study, using the $50 \mathrm{~K}$ version 2 for 2,226 bulls and the $50 \mathrm{~K}$ version 1 for 240 bulls. These 2,658 bulls built up the 50K Holstein Spanish population, which will be referred to as the $50 \mathrm{~K}$ population subsequently. Additionally, 192 bulls were genotyped using the 800K BovineHD BeadChip and will subsequently be referred as the HD population. These 2 sets have been contributed to the EuroGenomics (https://global.crv4all.com/ information/genomicselection/eurogenomics/) population. 
Table 1. Descriptors of traits considered in this study in terms of training and validation set sizes and ranges of heritability estimates

\begin{tabular}{lccc}
\hline Trait & $\begin{array}{c}\text { Training } \\
\text { set }\end{array}$ & $\begin{array}{c}\text { Validation } \\
\text { set }\end{array}$ & Heritability $^{1}$ \\
\hline Milk yield (MY) & 1,632 & 382 & $0.17-0.38$ \\
Fat percentage (FP) & 1,632 & 382 & $0.38-0.82$ \\
SCC & 1,629 & 380 & $0.16-0.18$ \\
Days open (DO) & 1,412 & 216 & $0.01-0.05$ \\
\hline
\end{tabular}

${ }^{1}$ Chauhan and Hayes (1991), Pösö and Mäntysaari (1996), and Pryce et al. (1997).

Before carrying out genome-based sire evaluations, SNP with greater than $5 \%$ incidence of missing genotypes across individuals, and SNP with minor allele frequency (MAF) less than 5\% were discarded, leaving 39,714 and 540,501 SNP for the 50K and HD evaluations, respectively. Animals with call rates lower than $90 \%$ were also excluded from the evaluation process.

Four complex traits of economic interest were examined, including milk yield (MY), fat percentage (FP), SCC, and days open (DO). These traits represent different heritabilities, genetic architectures, and amount of phenotypic information available (Table 1).

Deregressed multiple across-country evaluation (MACE) progeny proofs (DRP) from the January 2009 Interbull (Uppsala, Sweden) evaluation, calculated as described by Jairath et al. (1998), and genotypes from progeny-tested bulls in the training sets were used to estimate marker effects. Bulls in the testing sets had DGV in December 2011, based on 20 or more effective daughter contributions (EDC) each. Effective daughter contribution is a measure of information related to the reliability used in the prediction of breeding values, taking into account the number of daughters and their distribution over herds (Fikse and Banos, 2001).

\section{Imputation from $L D$ to $50 \mathrm{~K}$}

The design of reference and testing sets for imputation process and genomic evaluation is outlined in Figure 1 . The design of training and testing sets followed recommendations from Mäntysaari et al. (2010), but their recommended 4-yr gap between training and testing sets was reduced to $3 \mathrm{yr}$ due to the small size of the reference population, thereby leaving more bulls in the training set to enhance the accuracy of estimated DGV.

Training and testing data sets were generated from individuals genotyped with the $50 \mathrm{~K}$ chip based on year of birth. A total of 1,632 bulls born before 2006, with progeny test results in January 2009, were used as the training set for imputation and for genome-based evaluation of production traits (MY and FP). Then, 1,629 and 1,412 bulls were used as training populations for SCC and DO, respectively. Bulls born between 2006 and 2010 were used as the testing set, resulting in 834 animals with genotypes to be imputed, of which 382 were progeny-tested bulls in December 2011. These sires were used as the testing set for MY and FP genomic predictions, whereas only 380 and 216 had enough EDC for SCC and DO, respectively.

Low-density genotypes were created in silico in the testing set for its posterior assessment of imputation accuracy. For this purpose, SNP from the 50K assay that were not included in the GoldenGate Bovine3K BeadChip (LD3K; Illumina Inc.) or the BovineLD BeadChip (LD6K; Illumina Inc.) assays were masked, leaving 2,824 and 6,854 unmasked SNP, respectively. Thereafter, phased haplotypes from the reference population (TRAIN50K) filled in by BEAGLE were used as reference for imputation of the LD3K and LD6K validation sets, as well as for the imputation of missing SNP in the original $50 \mathrm{~K}$ set. The outcomes were referred as 3K50K, 6K50K, and TEST50K data sets.

\section{Imputation from 50K to HD}

The HD genotypes imputed from 3K50K, 6K50K, TEST50K, and TRAIN50K set were defined as 3KHD, 6KHD, 50KHD, and TRAIN50KHD respectively (Table 2), using the original HD population as the training set. Imputation to HD was performed in 2 steps for the 3KLD and 6KLD sets, as recommended in VanRaden et al. (2013).

\section{Accuracy of Imputation}

The accuracy of the imputation process was measured using the allele error rate (AER). The errors were counted as 0 when the imputed and actual marker genotypes were identical, 1 if the actual marker type was homozygous and the imputed genotype was heterozygous (or vice versa), and 2 if the actual and imputed marker types were homozygotes for distinct alleles. Markers/animals where the observed genotype was not missing in the original non-imputed data set were considered. The AER was calculated as 100 times the total number of errors divided by twice the number 


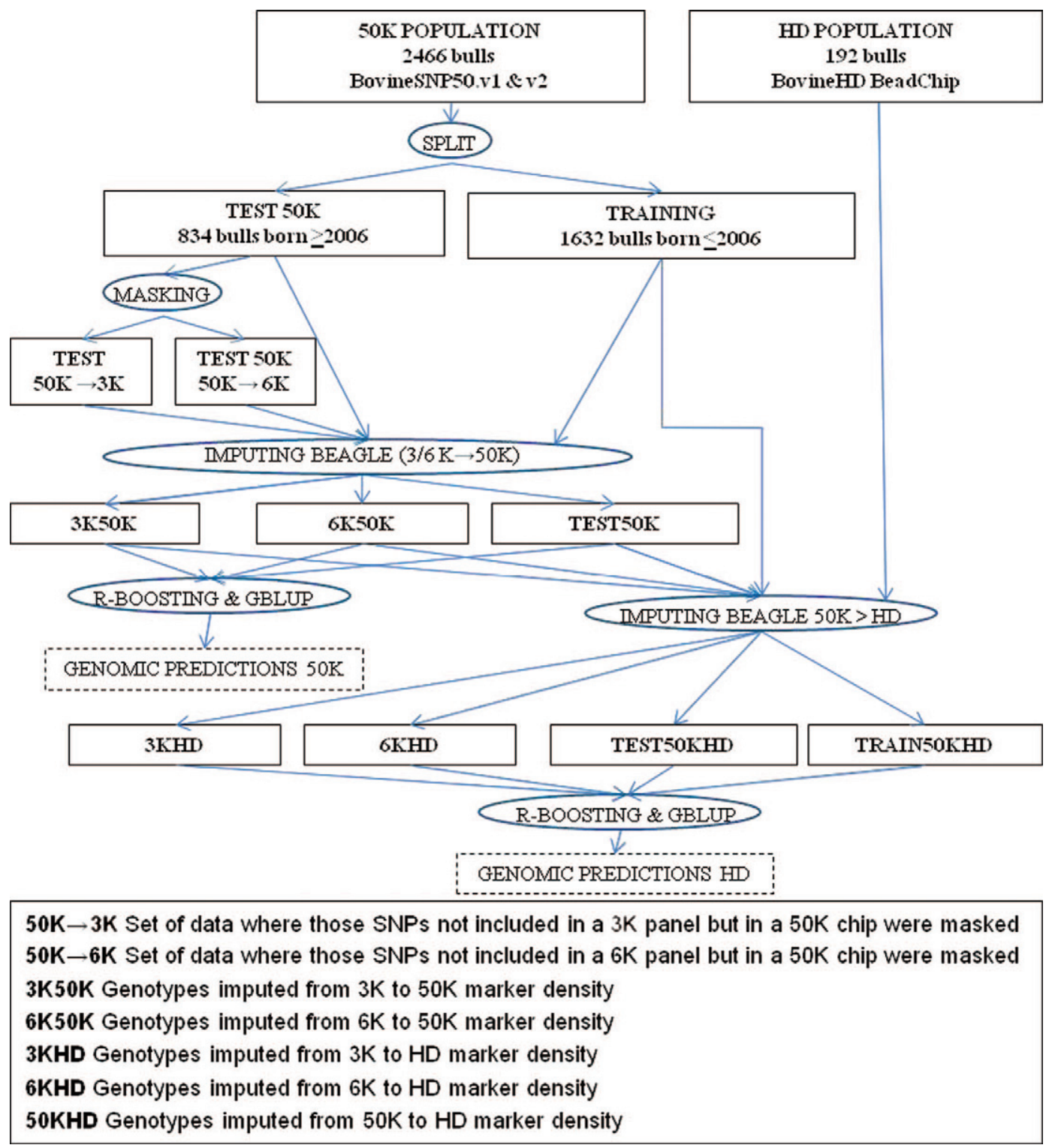

Figure 1. Diagram of the design of training and testing sets and process of masking, imputation, and genomic evaluations. $50 \mathrm{~K}=50,000$ markers; HD = high density; $3 \mathrm{~K}=3,000$ markers; $6 \mathrm{~K}=6,000$ markers; R-Boosting = random boosting; GBLUP = genomic BLUP; TRAIN50K $=$ reference population; BovineSNP50.v1 \& v2 BeadChip $=50 \mathrm{~K}$ panels by Illumina Inc. (San Diego, CA); BovineHD BeadChip = 800,000-SNP panel by Illumina Inc. Color version available in the online PDF.

of imputed loci. This gave the rate of falsely predicted alleles, which is an appropriate measure when using an additive prediction model, as in this study. Allele error rate can be approximated as half the genotype error rate (Druet et al., 2010).

\section{Genome-Enabled Evaluation Models}

It should be noted that the original HD population was used only for imputation purposes, and genomebased evaluation comparisons were carried out between imputed $50 \mathrm{~K}$ and $800 \mathrm{~K}$ genotypes with the original $50 \mathrm{~K}$ data set. Two different evaluation models were used.
$\boldsymbol{R}$-Boost. The random boosting algorithm (González-Recio et al., 2013) is a machine-learning technique that combines different predictors and a shrinkage factor (Friedman, 2000). Boosting adds basis functions iteratively, such that each addition further reduces the selected loss function (Hastie et al., 2005). Ordinary least squares estimation with genotypes as basis function was successively applied on the residuals of a previous iteration in the reference population. The mean squared error (MSE) of prediction in a tuning set was used as the loss function to be minimized. The marker effect shrinkage parameter $v$ of the algorithm 
Table 2. Descriptors of original information, marker density modification process, and final marker density for each considered data set included in the study ${ }^{1}$

\begin{tabular}{|c|c|c|c|}
\hline Data set & $\begin{array}{l}\text { Original } \\
\text { data set }\end{array}$ & $\begin{array}{l}\text { Final } \\
\text { density }\end{array}$ & Process \\
\hline TEST50K & TEST50K & $50 \mathrm{~K}$ & \\
\hline TRAIN50K & TRAIN50K & $50 \mathrm{~K}$ & \\
\hline $3 \mathrm{~K} 50 \mathrm{~K}$ & TEST50K & $50 \mathrm{~K}$ & $\begin{array}{l}\text { Masking } 50 \mathrm{~K} \rightarrow 3 \mathrm{~K} \\
\text { Imputation } 3 \mathrm{~K} \rightarrow 50 \mathrm{~K}\end{array}$ \\
\hline $6 \mathrm{~K} 50 \mathrm{~K}$ & TEST50K & $50 \mathrm{~K}$ & $\begin{array}{l}\text { Masking } 50 \mathrm{~K} \rightarrow 6 \mathrm{~K} \\
\text { Imputation } 6 \mathrm{~K} \rightarrow 50 \mathrm{~K}\end{array}$ \\
\hline 3KHD & $3 \mathrm{~K} 50 \mathrm{~K}$ & HD & Imputation $50 \mathrm{~K} \rightarrow \mathrm{HD}$ \\
\hline $6 \mathrm{KHD}$ & $6 \mathrm{~K} 50 \mathrm{~K}$ & HD & Imputation $50 \mathrm{~K} \rightarrow \mathrm{HD}$ \\
\hline TEST50KHD & TEST50K & HD & Imputation $50 \mathrm{~K} \rightarrow \mathrm{HD}$ \\
\hline TRAINHD & TRAIN50K & $\mathrm{HD}$ & Imputation $50 \mathrm{~K} \rightarrow \mathrm{HD}$ \\
\hline
\end{tabular}

${ }^{1} 50 \mathrm{~K}=50,000$ markers; $3 \mathrm{~K}=3,000$ markers; $6 \mathrm{~K}=6,000$ markers; HD $=$ high density.

was fixed at 0.10 , whereas the parameter mtry that sets the percentage of markers sampled per iteration was fixed at 0.05 , as suggested by González-Recio et al. (2013).

$\boldsymbol{G}$-BLUP. The G-BLUP method is similar to standard BLUP evaluations, with the pedigree relationship matrix replaced with a genomic relationship matrix (G) built from molecular information. Pairs of individuals sharing the same genotype for a large number of markers will be more similar genomically, and will have larger values in the corresponding off-diagonal cells of the matrix, as is the case for pairs of related animals in a pedigree-based relationship matrix. The genomic relationship matrix was computed as $\mathbf{G}=\frac{\mathbf{Z Z}^{\prime}}{2 \sum p_{i}\left(1-p_{i}\right)}$, following VanRaden (2008), where $p$ is the minor allele frequency of locus $i$; $\mathbf{Z}$ is a matrix that results from subtracting $\mathrm{P}$ from $\mathbf{M}$, with $\mathrm{P}=2\left(p_{i}\right.$ $-0.5)$, where $p_{i}$ is the frequency of the second allele at locus $i$; and $\mathbf{M}$ is the matrix of genotypes coded as -1 , 0 , and 1 for the homozygote, heterozygote, and other homozygote, respectively.

\section{Criteria for Comparisons}

Reliability, Empirical Bias in Regression Coefficients, and MSE. The prediction accuracy of evaluations was computed as weighted by EDC Pearson correlation between the predicted DGV in the testing set and the December 2011 DRP. Regression coefficients of the realized DRP on the estimated DGV were also calculated, because this parameter is commonly used as a measure of prediction bias in genome-assisted evaluations (Mäntysaari et al., 2010). Finally, the MSE of predictions was also estimated.

Means and confidence intervals were estimated using bootstrapped samples in each evaluation output (Efron and Tibshirani, 1986). Pairs were the predicted phenotype in the testing set and its corresponding observed (known) phenotype. Then, 1,000 samples were drawn with replacement from the whole testing set, and predictive correlation estimates, regression coefficients and MSE correlation were computed for each of the bootstrap samples. The MSE is preferable as criterion when animals with different amounts of information are compared (Vitezica et al., 2011). Finally, the confidence interval was considered as the narrowest interval, containing $95 \%$ of the replicates.

Selection Effectiveness. Selection effectiveness was measured as $\alpha_{\text {top }} / \alpha_{\text {sel }}$, where $\alpha_{\text {sel }}$ represents a given percentage of bulls ranked by their predicted DGV and $\alpha_{\text {top }}$ represents the percentage of bulls selected by the model that were in the same percentile according to their realized DPR. This measure can be interpreted as the fraction of young bulls as ranked by DGV that actually included at least 1 truly top bull, or similarly, as the fraction of truly top bulls that was included in a given set of top young bulls as predicted by DGV.

Selection effectiveness can also be measured using confusion matrices. These matrices are commonly used in classification problems (Edler et al., 2001), and are built by comparing predictions with realizations in a validation data set. In the current study, predictions and observations across traits and methods were split into 5 disjoint classes according to their observed DRP and predicted DGV rankings. Therefore, each class included $20 \%$ of bulls in the testing set. Observations were classified in rows and predictions in columns. Correct predictions fall on the diagonals $\left(a_{i i}\right)$, and misclassifications on the off-diagonal $\left(a_{i j}, i \neq j\right)$ of the confusion matrix. Elements above the diagonal represent bulls that were underevaluated by the genomic method, whereas outcomes below the diagonal represent bulls that were overevaluated. Confusion matrices allow computation of overall selection effectiveness $(E)$ as the proportion of correctly classified observations for a given $\mathbf{C}$ matrix: 


$$
E=\frac{\sum_{i=1}^{n} \sum_{j=1}^{m} c_{i j},(i=j)}{\sum_{i=1}^{n} \sum_{j=1}^{m} c_{i j}},
$$

where $c_{i i}$ are the elements on the diagonal. Dairy cattle breeding programs may be interested on the effectiveness of top bulls selection; this can be computed from the confusion tables as the proportion of correctly classified bulls in the first class: $E_{t o p}=\frac{c_{11}}{\sum_{i=1}^{n} \sum c_{i 1}}$. Similarly, effectiveness of selection was also computed for the top $60 \%$ bulls to provide a measure at low selectionintensity scenarios:

$$
E_{60 \%}=\frac{\sum_{i=1}^{3} \sum_{j=1}^{3} c_{i j}}{\sum_{i=1}^{n} \sum_{j=1}^{3} c_{i j}} .
$$

\section{RESULTS AND DISCUSSION}

\section{Imputation Performance}

Imputation performances from customized LD3K and LD6K to 50K density resulted in an AER of 3.1 and $1.3 \%$, respectively. These results agree with previous studies using similar population sizes (Zhang and Druet, 2010; Berry and Kearney, 2011; Dassonneville et al., 2012). Based on these results, use of the LD6K array should be an important improvement.

It must be noted that our results may be slightly overoptimistic, because LD genotypes are masked instead of directly genotyped, especially in the case of $3 \mathrm{KLD}$, due to the different chemistries used (Dassonneville et al., 2012). Regarding imputation from $50 \mathrm{~K}$ to $\mathrm{HD}$, a small number of HD genotypes could be enough for accurate imputation in some populations [C. Schrooten (CRV BC, Arnhem, the Netherlands), R. Dassonneville (INRA, GABI, Jouy-en-Josas, France), R. Brøndum (Department of Molecular Biology and Genetics, Tjele, Denmark), J. Chen (VIT, Verden, Germany), Z. Liu (VIT), and T. Druet (Unit of Animal Genomics, Faculty of Veterinary Medicine and Centre for Biomedical Integrative Genoproteinomics, University of Liège, Liège, Belgium), personal communication], despite the fact that this accuracy can be enhanced when more HD genotypes are included within the range of genotypes used in the current study. In a previous pilot study, AER $(\times 100)$ after imputation from 50K to HD was $0.9 \%$ for our set of data.

\section{Validation of Genome-Enabled Evaluations}

Reliability. Table 3 shows the results for predictive reliability obtained with the 2 methods considered.
Both methods resulted in similar accuracy; R-Boost was the preferred method for FP, whereas G-BLUP was preferred for MY and DO. No clear advantages for a particular method were observed for SCC.

Regarding SNP density, the imputation of LD genotypes to a $50 \mathrm{~K}$ panel resulted in accuracies in the range to these observed for the original $50 \mathrm{~K}$ genotypes, mainly for the LD6K case, in agreement with results reported previously by Segelke et al. (2012). Predictive reliability from $6 \mathrm{~K}$ to $50 \mathrm{~K}$ was $2 \%$ higher, averaged across traits, than from 3K. Because the LD3K assay was developed using a different technology than the $6 \mathrm{~K}$ and $50 \mathrm{~K}$ panels, differences are expected to be larger when real data instead of masked genotypes are used (Dassonneville et al., 2012). For traits related to fertility, imputed genotypes were competitive compared with the $50 \mathrm{~K}$ data set.

Lower reliability for MY using genotypes imputed to $\mathrm{HD}$ was found regarding the original 50K genotypes. However, modest improvements occurred for FP, SCC, and DO. Evaluation of genotypes imputed to HD was on average, across data set, method, and trait, $4 \%$ more accurate than the $50 \mathrm{~K}$ prediction. Similar performance was found between data sets imputed from LD to HD. This result was in accordance with those previously reported in other Holstein populations, where HD estimates were only slightly better than those from $50 \mathrm{~K}$ genotypes (Erbe et al., 2012; VanRaden et al., 2013). In a recent study, Jensen et al. (2012) concluded that $92 \%$ of all additive genetic variance could be explained using 44,000 (44K) SNP markers, and that further increases in marker density would have limited effects on the predictive accuracy, unless better methods are used to distinguish between markers with real effects and markers with no effect.

Confidence intervals estimated by bootstrapping showed that distributions of prediction reliability widely overlapped across methods and sets for MY and SCC. However, R-Boost estimates were more accurate than G-BLUP for FP. As expected, large confidence intervals were found for DO, probably due to the reference population size and the amount of information for each bull. The aim of inclusion of those confidence intervals was to show which differences were noticeable and which can be associated with the level of uncertainty associated with our data.

Some results were quite surprising, such as the fact that 3 out of 8 6KHD predictions were higher than the respective 50KHD; however, taking into account confidence intervals, those were almost identical. Uncertainty associated with those predictions was larger than differences between cases.

Larger uncertainty was associated with DO results due to the lower heritability of the trait and limited 
amount of information. Consequently, unexpected results were found as in the case of low Pearson correlations of TEST50K G-BLUP predictions. Nevertheless, the value of 0.19 is within all confidence intervals calculated for this trait and methods and should be considered plausible.

Empirical Bias of Regression Coefficients. The estimated slope coefficients (b values) of the regression of realized DRP on estimated DGV for the traits, methods, and genotyped sets considered are shown in Table 4. Estimated regression coefficients were close to unity for MY, SCC, and DO using R-Boost, whereas GBLUP showed values closer to unity for FP. These values were within the range of previously reported values for other populations (Olson et al., 2011). No relevant differences were found between 3KLD and 6KLD in terms of the $\mathrm{b}$ values. In general, data sets using HD genotypes resulted in similar departures from unity as the evaluations using $50 \mathrm{~K}$ genotypes. However, for DO, the imputation to HD led to more favorable $\mathrm{b}$ values but these were still far from the unity when G-BLUP was used. Su et al. (2012) also reported slightly better performance at HD density for fertility traits regarding bias in the regression coefficient. Values of the coefficient for this case should increase if new animals are included in the reference population and their DRP are based on larger EDC. Confidence intervals from R-Boost included the unity for MY in the $50 \mathrm{~K}$ data set, and all data sets for SCC and DO, the latter as a result of a large uncertainty. However, for G-BLUP only estimates for FP included unity.

$\boldsymbol{M S E}$. The MSE of predictions showed differences between evaluation methods (Table 5 ). Random boosting had smaller MSE for the 4 traits, although confidence intervals overlapped for all traits except for FP, where R-Boost showed significantly smaller MSE. In general, MSE estimates from R-Boost predictions were $12,54,12$, and $5 \%$ smaller than those from G-BLUP for MY, FP, SCC, and DO, respectively. These results and the aforementioned reliabilities and regression coefficients are in agreement with those reported by Jiménez-Montero et al. (2013) who compared different methods using a similar population and different traits.

The 6KLD set showed smaller MSE than 3KLD for $\mathrm{MY}$ and FP after imputation, but no clear differences were found for SCC and DO. Slightly smaller MSE was observed for the 50KHD set, regardless of the trait and method, except for MY, and the improvement averaged only $1 \%$ across traits. The $50 \mathrm{KHD}$ set also outperformed LD sets after imputation to HD. Imputation from $50 \mathrm{~K}$ to $\mathrm{HD}$ resulted in 1 and $3 \%$ smaller MSE averaged across traits and methods regarding imputation from $6 \mathrm{~K}$ and $3 \mathrm{~K}$, respectively. The MSE of $\mathrm{HD}$ 
T) Table 4. Regression coefficients from genomic BLUP (G-BLUP) and random boosting (R-Boost) for 4 traits after imputation from 3,000 markers (3K), 6,000 markers (6K), and 50,000 markers $(50 \mathrm{~K})$ to $50 \mathrm{~K}$ and high density $(\mathrm{HD})^{1}$

\begin{tabular}{|c|c|c|c|c|c|c|c|}
\hline Trait & Method & $3 \mathrm{~K} 50 \mathrm{~K}$ & $6 \mathrm{~K} 50 \mathrm{~K}$ & TEST50K & $3 \mathrm{KHD}$ & $6 \mathrm{KHD}$ & $50 \mathrm{KHD}$ \\
\hline \multirow[t]{2}{*}{ Milk yield (MY) } & G-BLUP & $0.74(0.63-0.84)$ & $0.72(0.61-0.82)$ & $0.72(0.62-0.83)$ & $0.68(0.57-0.79)$ & $0.67(0.56-0.76)$ & $0.67(0.58-0.79)$ \\
\hline & R-Boost & $0.86(0.72-1.01)$ & $0.88(0.75-1.03)$ & $\mathbf{0 . 9 0}(0.77-1.05)$ & $0.84(0.71-0.99)$ & $0.85(0.69-0.97)$ & $0.85(0.70-0.98)$ \\
\hline \multirow{2}{*}{ Fat percentage (FP) } & G-BLUP & $1.08(0.94-1.23)$ & $1.02(0.88-1.15)$ & $1.01(0.87-1.14)$ & $0.93(0.78-1.08)$ & $0.94(0.80-1.11)$ & $0.93(0.78-1.08)$ \\
\hline & R-Boost & $1.17(1.05-1.28)$ & $1.29(1.19-1.40)$ & $1.30(1.20-1.41)$ & $1.13(1.01-1.22)$ & $1.24(1.15-1.34)$ & $1.25(1.15-1.36)$ \\
\hline \multirow[t]{2}{*}{$\mathrm{SCC}$} & G-BLUP & $0.59(0.48-0.73)$ & $0.60(0.47-0.72)$ & $0.58(0.45-0.69)$ & $0.62(0.47-0.78)$ & $0.61(0.49-0.74)$ & $0.67(0.51-0.81)$ \\
\hline & R-Boost & $1.03(0.79-1.27)$ & $0.98(0.76-1.23)$ & $1.00(0.77-1.22)$ & $1.03(0.79-1.29)$ & $1.06(0.83-1.26)$ & $1.03(0.82-1.25)$ \\
\hline \multirow{2}{*}{ Days open (DO) } & G-BLUP & $0.39(0.14-0.64)$ & $0.45(0.21-0.70)$ & $0.25(0.03-0.45)$ & $0.47(0.21-0.70)$ & $0.51(0.28-0.78)$ & $0.51(0.26-0.75)$ \\
\hline & R-Boost & $0.74(0.15-1.43)$ & $0.64(0.10-1.14)$ & $\mathbf{0 . 9 9}(0.31-1.70)$ & $0.85(0.30-1.33)$ & $0.58(0.14-1.06)$ & $0.91(0.35-1.44)$ \\
\hline
\end{tabular}

${ }^{1}$ Mean of the 1,000 bootstrap replicates and bootstrap CI (in parentheses) constructed as the narrowest gap, containing $95 \%$ of the replicates, are shown. The preferred methods within trait and set criterion are in bold.

Table 5. Mean squared errors for genomic BLUP (G-BLUP) and random boosting (R-Boost) for 4 traits after imputation from 3,000 markers (3K), 6,000 markers (6K), and 50,000 markers $(50 \mathrm{~K})$ to $50 \mathrm{~K}$ and high density $(\mathrm{HD})^{1}$

\begin{tabular}{|c|c|c|c|c|c|c|c|}
\hline Trait & Method & $3 \mathrm{~K} 50 \mathrm{~K}$ & $6 \mathrm{~K} 50 \mathrm{~K}$ & TEST50K & $3 \mathrm{KHD}$ & $6 \mathrm{KHD}$ & $50 \mathrm{KHD}$ \\
\hline \multirow[t]{2}{*}{ Milk yield $(\mathrm{MY})^{2}$} & G-BLUP & $256(217-291)$ & $255(220-296)$ & $258(222-299)$ & $277(237-317)$ & $276(240-317)$ & $278(238-315)$ \\
\hline & R-Boost & $244(206-280)$ & $236(201-274)$ & $229(198-266)$ & $247(212-284)$ & $241(206-278)$ & $240(205-281)$ \\
\hline \multirow{2}{*}{ Fat percentage $(\mathrm{FP})$} & G-BLUP & $0.044(0.038-0.051)$ & $0.044(0.038-0.050)$ & $0.044(0.039-0.051)$ & $0.048(0.042-0.055)$ & $0.048(0.041-0.054)$ & $0.047(0.041-0.054)$ \\
\hline & R-Boost & $0.034(0.028-0.039)$ & $0.030(0.026-0.034)$ & $0.030(0.026-0.035)$ & $0.032(0.026-0.038)$ & $0.028(0.024-0.032)$ & $\mathbf{0 . 0 2 7}(0.023-0.032)$ \\
\hline \multirow[t]{2}{*}{$\mathrm{SCC}$} & G-BLUP & $157.8(134.5-178.9)$ & $155.1(130.2-175.7)$ & $154.5(132.3-177.4)$ & $149.2(126.7-171.3)$ & $152.0(129.1-172.2)$ & $143.3(122.9-164.5)$ \\
\hline & R-Boost & $136.6(117.5-157.7)$ & $138.3(118.8-160.5)$ & $137.4(115.4-158.0)$ & $137.1(117.8-156.4)$ & $131.7(115.0-153.6)$ & $133.4(114.2-154.5)$ \\
\hline \multirow{2}{*}{ Days open (DO) } & G-BLUP & $562.6(460.1-673.3)$ & $548.6(446.4-656.1)$ & $636.3(518.8-758.7)$ & $537.5(433.5-642.0)$ & $530.2(437.5-638.8)$ & $535.1(440.1-644.1)$ \\
\hline & R-Boost & $531.7(429.6-638.2)$ & $541.9(441.1-641.5)$ & $523.4(429.5-634.7)$ & $\mathbf{5 1 9 . 4}(405.7-618.7)$ & $546.4(426.5-650.9)$ & $519.6(415.7-619.2)$ \\
\hline
\end{tabular}

${ }^{1}$ Mean of the 1,000 bootstrap replicates and bootstrap CI (in parentheses) constructed as the narrowest gap, containing $95 \%$ of the replicates, are shown. The preferred methods within trait and set criterion are shown in bold.

${ }^{2}$ Values $\times 1,000$. 
prediction was, on average across data set, method, and trait, $2 \%$ lower than the $50 \mathrm{~K}$ prediction.

Bootstrap confidence intervals from G-BLUP and R-Boost overlapped for MY, SCC, and DO, showing similar levels of uncertainty. Random boosting showed clearly smaller MSE for FP as reported above for reliability and regression slopes; estimations with 50K sets were preferred over HD for MY. Nevertheless, MSE from predictions using HD genotypes was smaller for FP, SCC, and DO, but no clear differences were obtained and the bootstrapped confidence intervals overlapped.

Some results could be confusing, as it seems that imputation could improve accuracy in some cases. However, it is not possible to state such a conclusion from our results, but only that the effect of imputation is limited in terms of accuracy, bias, and MSE. Those unexpected differences were always within the limits of the calculated confidence interval and could be associated with the level of uncertainty of the set of data used.

Selection Effectiveness. Both R-Boost and G-BLUP performed in a similar manner at selecting top-ranked bulls regarding their observed DRP (Supplemental Figure S1; http://dx.doi.org/10.3168/ jds.2013-6793). Differences were observed only for FP in favor of R-Boost (Figure 2). Selection accuracy of top bulls was slightly higher with HD genotypes; as

\section{G-BLUP FP}

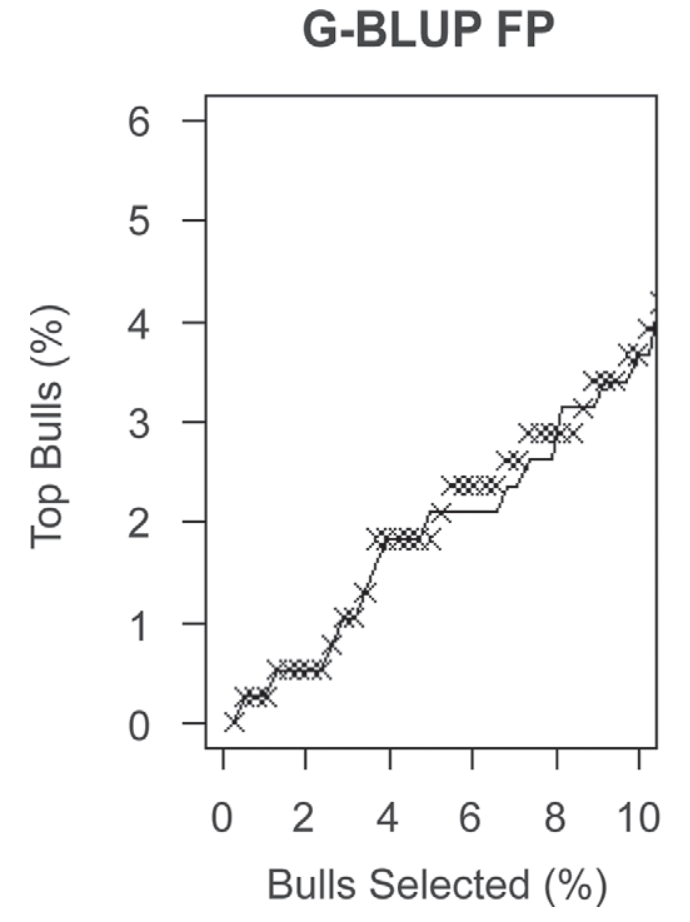

an example, $57 \%$ instead of $50 \%$ of bulls ranked in the 90th percentile regarding observed DRP were also ranked in the 90th percentile regarding DGV. A large percentage of actual top bulls for MY, FP, and DO were ranked on top when R-Boost was the evaluation method and HD genotypes were used. For other cases, $50 \mathrm{~K}$ and HD resulted in similar patterns. The R-Boost showed better selection effectiveness than G-BLUP using HD genotypes, probably because R-Boost is based on estimation of individual SNP effects instead of average genomic similarity between pairs of individuals.

The trait FP showed clear differences across methods for all considered criteria; this performance has been previously reported (Jiménez-Montero et al., 2013). Methods based on genomic relationships seem not able to capture all the information provided by the data when the reference population is limited. An explanation of this behavior is commonly related to the larger effect of a single gene (DGAT1) controlling the trait (Daetwyler et al., 2010).

Another way to measure selection efficiency is through confusion tables. Bulls in the testing sets were classified according to observed DRPs and predicted DGV rankings (Supplemental Table S1; http://dx.doi. org/10.3168/jds.2013-6793). An equal or larger number of animals in each percentile were correctly classified regarding observed DRP using imputation from $50 \mathrm{~K}$ to

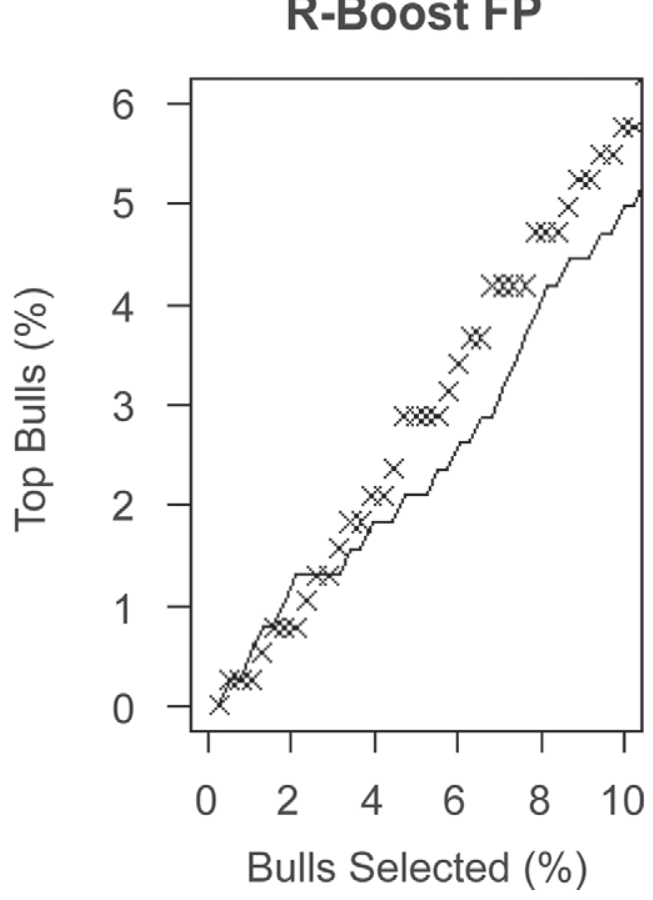

Figure 2. Percentage of common bulls in the observed and predicted rankings when top $10 \%$ or less of the genomically evaluated bulls are selected for fat percentage (FP). Comparison between 50,000-marker (50K; - ) and high-density (HD; × ) genotypes. G-BLUP = genomic BLUP; $\mathrm{R}$-Boost $=$ random boosting. 
HD than imputation from $6 \mathrm{~K}$ to $50 \mathrm{~K}$. Averaged across methods, 3 out of $382,8.5$ out of $382,0.5$ out of 380 , and 3.5 out of 216 more animals were classified in the same percentile for MY, FP, SCC, and DO respectively. For all cases, genomic evaluation methods classified correctly more animals in the 2 extreme classes than in the 3 intermediate. It should be noted that the original continuous variables were changed to categories, and the number of defined categories may affect the results.

Results from confusion matrices are also shown in Table 6 as rates, including overall rate of correctly classified animals, rate of correctly classified in the first class (top 20\%), and rate of correctly classified within the top 3 classes (top 60\%). Small differences were found between data sets and methods. Averaged across sets, R-Boost classified correctly more animals in the first class (top 20\%) when MY (43.5 vs. $42.8 \%$ ) and FP (56.3 vs. $48.8 \%$ ) were considered. However, GBLUP outperformed R-Boost for SCC (44.8 vs. $40.3 \%$ ) and DO (29.6 vs. 27.3\%). Also, for 3 out of 4 traits, FP (53.5 vs. $51.6 \%$ ), SCC (44.5 vs. $40.6 \%$ ), and DO (26.2 vs. $27.8 \%$ ) HD estimates correctly classified more animals as belonging to the top class than predictions based on $50 \mathrm{~K}$ genotypes. The rate of animals correctly classified using HD genotypes ranged between 0.21 for DO to 0.46 for FP, and it was only poorer for MY (41.6 vs. $44.6 \%)$. On average, $32 \%$ of animals were correctly classified regarding their observed DRP across traits, sets and methods. It must be noted that 20,20, and $60 \%$ of animals will be correctly classified if selected at random regarding overall, top 20\%, and top $60 \%$ criteria. From this point of view, GS increased these figures by 12,22 , and $13 \%$, respectively, and the use of GS seems to offer some advantages in high-selectionintensity scenarios. All predictive ability measures are based on DPR observations, which are approximations of the true breeding values and, therefore, some error is expected on the true classification of methods and sets.

\section{CONCLUSIONS}

The BEAGLE software was efficient for the reconstruction of $50 \mathrm{~K}$ genotypes from $\mathrm{LD}$ chips and also for imputation to HD, even with a small HD reference population. Genomic evaluation methods (R-Boost and G-BLUP) resulted in similar prediction ability for the traits and genotypes included in this study. R-Boost showed clearly better performance for specific traits. Genotypes in 3K density showed worse imputationaccuracy performance but only slightly worse predictive ability. In general, genotypes imputed from LD presented similar predictive ability to that from the

Table 6. Rate of animals correctly classified according to their ranking in 5 classes, each one containing $20 \%$ of the values (overall), correctly classified in the first class (top 20\%), or within the 3 highest classes (top 60\%) ${ }^{1}$

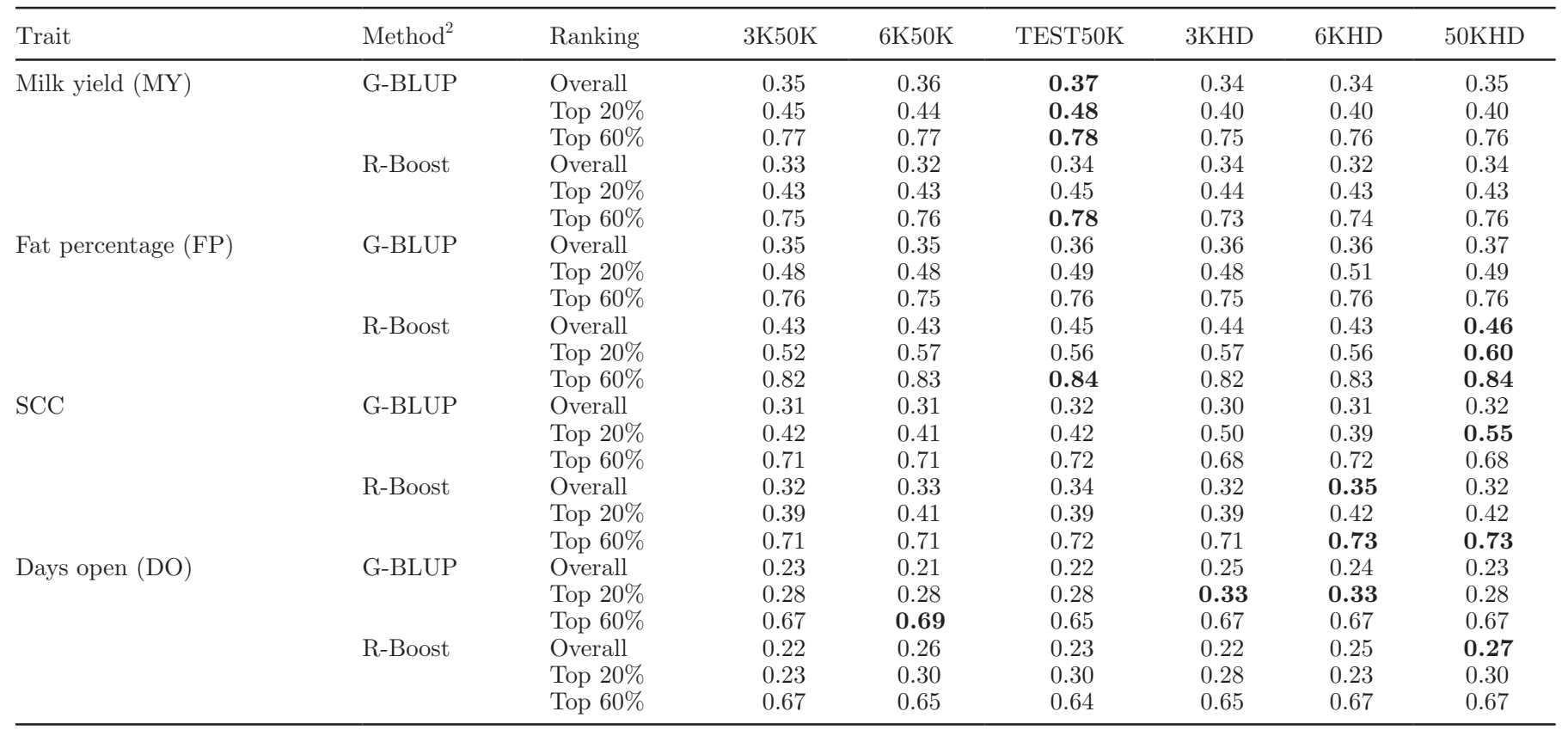

${ }^{1}$ Animals were ranked regarding observed deregressed multiple across-country evaluation (MACE) progeny proofs (DRP) for 4 traits and the evaluation methods used after imputation from 3,000 markers (3K), 6,000 markers (6K), and 50,000 markers (50K) to 50K and high density (HD). The preferred methods within trait and set criterion for the correct classification of bulls (overall), correct classification in the first class (top 20\%), or within 3 top classes (top 60\%) are shown in bold.

${ }^{2} \mathrm{G}-\mathrm{BLUP}=$ genomic BLUP; R-Boost $=$ random boosting. 
original $50 \mathrm{~K}$ genotypes. However, imputation to HD showed $2 \%$ smaller MSE of yet-to-be observed DPR, providing an interesting alternative to reduce bias of predictions, and more stability in the genomic proofs of young candidates. Low-density genotyping and posterior imputation is an interesting approach to reduce genotyping costs for preselection of young candidates and on-farm decisions, as no remarkable lack on selection efficiency is caused during the imputation process. Selection efficiency could be slightly enhanced for certain traits, such as FP, SCC, or DO, especially when the aim of the evaluation is to detect top animals in the population. Genetic evaluation units may consider a trait-dependent strategy in terms of method and genotype density for their use in the genome-enhanced evaluations. Traits such as FP showed clear differences across methods; also, genotype density produced unequal effects on posterior genome-enhanced evaluations.

\section{ACKNOWLEDGMENTS}

We especially thank R. Dassonneville for helpful suggestions and comments. The authors acknowledge funds from project CDTI-P080250866 UPM and agreement INIA-CC10-046 [Instituto Nacional de Investigación y Tecnología Agraria y Alimentaria (INIA), Madrid, Spain], and thank Confederación de Asociaciones de Frisona Española (CONAFE; Madrid, Spain), EuroGenomics Consortium, Ascol (Asturias, Spain), Aberekin SA (Derio, Spain), Xenética Fontao SA (Lugo, Spain), and Genetical (Avila, Spain) for providing biological samples and phenotypes used in this study. The authors also express gratitude to Dirección General de Producciones y Mercados Agrarios, Laboratorio Central de Veterinaria del Ministerio de Agricultura, Alimentación y Medio Ambiente (Madrid, Spain) for support during the genotyping process.

\section{REFERENCES}

Berry, D. P., and J. F. Kearney. 2011. Imputation of genotypes from low- to high-density genotyping platforms and implications for genomic selection. Animal 5:1162-1169.

Boichard, D., H. Chung, R. Dassonneville, X. David, A. Eggen, S. Fritz, K. J. Gietzen, B. J. Hayes, C.T. Lawley, T. S. Sonstegard, C. P. Van Tassell, P. M. VanRaden, K. A. Viaud-Martinez, and G. R. Wiggans., and the Bovine LD Consortium. 2012. Design of a bovine low-density SNP array optimized for imputation. PLoS ONE 7:e34130.

Browning, B. L., and S. R. Browning. 2009. A unified approach to genotype imputation and haplotype-phase inference for large data sets of trios and unrelated individuals. Am. J. Hum. Genet. 84:210-223.

Calus, M. P. L., R. F. Veerkamp, and H. A. Mulder. 2011. Imputation of missing single nucleotide polymorphism genotypes using a multivariate mixed model framework. J. Anim. Sci. 89:2042-2049.

Chauhan, V. P. S., and J. F. Hayes. 1991. Genetic parameters for first lactation milk production and composition traits for Holsteins using multivariate restricted maximum likelihood. J. Dairy Sci. 74:603-610.

Daetwyler, H. D., R. Pong-Wong, B. Villanueva, and J. A. Woolliams. 2010. The impact of genetic architecture on genome-wide evaluation methods. Genetics 185:1021-1031. http://dx.doi. org/10.1534/genetics.110.116855.

Dassonneville, R., S. Fritz, V. Ducrocq, and D. Boichard. 2012. Short communication: Imputation performances of 3 low-density marker panels in beef and dairy cattle. J. Dairy Sci. 95:4136-4140.

de los Campos, G., H. Naya, D. Gianola, J. Crossa, A. Legarra, E. Manfredi, K. Weigel, and J. M. Cotes. 2009. Predicting quantitative traits with regression models for dense molecular markers and pedigree. Genetics 182:375-385.

de Roos, A. P. W., C. Schrooten, E. Mullaart, S. van der Beek, G. de Jong, and W. Voskamp. 2009. Genomic selection at CRV. Interbull Bull. 39:47-50.

Druet, T., C. Schrooten, and A. P. W. de Roos. 2010. Imputation of genotypes from different single nucleotide polymorphism panels in dairy cattle. J. Dairy Sci. 93:5443-5454.

Edler, L., J. Grassmann, and S. Suhai. 2001. Role and results of statistical methods in protein fold class prediction. Math. Comput. Model. 33:1401-1417.

Efron, B., and R. Tibshirani.. 1986. Bootstrap methods for standard errors, confidence intervals, and other measures of statistical accuracy. Stat. Sci. 1:54-75.

Erbe, M., B. J. Hayes, L. K. Matukumalli, S. Goswami, P. J. Bowman, C. M. Reich, B. A. Mason, and M. E. Goddard. 2012. Improving accuracy of genomic predictions within and between dairy cattle breeds with imputed high-density single nucleotide polymorphism panels. J. Dairy Sci. 95:4114-4129.

Fikse, W. F., and G. Banos. 2001. Weighting factors of sire daughter information in international genetic evaluations. J. Dairy Sci. 84:1759-1767.

Friedman, J. H., 2000. Greedy function approximation: A gradient boosting machine. Ann. Statist. 29:1189-1232.

González-Recio, O., J. A. Jiménez-Montero, and R. Alenda. 2013. The gradient boosting algorithm and random boosting for genomeassisted evaluation in large data sets. J. Dairy Sci. 96:614-624.

Hao, K., E. Chudin, J. McElwee, and E. Schadt. 2009. Accuracy of genome-wide imputation of untyped markers and impacts on statistical power for association studies. BMC Genet. 10:27.

Harris, B. L., D. L. Johnson, and R. J. Spelman. 2008. Genomic selection in New Zealand and the implications for national genetic evaluation. Proceedings of the 36th International Committee for Animal Recording Biennial Session, Niagara Falls. ICAR Technical Series, 13:325-330.

Hastie, T., R. Tibshirani, J. Friedman, and J. Franklin. 2005. The elements of statistical learning: Data mining, inference and prediction. The Mathematical Intelligencer 27:83-85. http://dx.doi. org/10.1007/BF02985802.

Howie, B. N., P. Donnelly, and J. Marchini. 2009. A flexible and accurate genotype imputation method for the next generation of genome-wide association studies. PLoS Genet. 5:e1000529.

Jairath, L., J. C. M. Dekkers, L. R. Schaeffer, Z. Liu, E. B. Burnside, and B. Kolstad. 1998. Genetic evaluation for herd life in Canada. J. Dairy Sci. 81:550-562.

Jensen, J., G. Su, and P. Madsen. 2012. Partitioning additive genetic variance into genomic and remaining polygenic components for complex traits in dairy cattle. BMC Genet. 13:44.

Jiménez-Montero, J. A., O. González-Recio, and R. Alenda. 2013. Comparison of methods for the implementation of genome-assisted evaluation of Spanish dairy cattle. J. Dairy Sci. 96:625-634.

Kong, A., G. Masson, M. L. Frigge, A. Gylfason, P. Zusmanovich, G. Thorleifsson, P. I. Olason, A. Ingason, S. Steinberg, T. Rafnar, P. Sulem, M. Mouy, F. Jonsson, U. Thorsteinsdottir, D. F. Gudbjartsson, H. Stefansson, and K. Stefansson. 2008. Detection of sharing by descent, long-range phasing and haplotype imputation. Nat. Genet. 40:1068-1075.

Long, N., D. Gianola, G. J. M. Rosa, K. A. Weigel, and S. Avendaño. 2007. Machine learning classification procedure for selecting SNPs 
in genomic selection: application to early mortality in broilers. J. Anim. Breed. Genet. 124:377-389.

Mäntysaari, E., Z. Liu, and P. VanRaden. 2010. Validation test for genomic evaluations. Interbull Bull. 41:17-22.

Meuwissen, T., and M. Goddard. 2010. The use of family relationships and linkage disequilibrium to impute phase and missing genotypes in up to whole-genome sequence density genotypic data. Genetics 185:1441-1449.

Meuwissen, T. H. E., B. J. Hayes, and M. E. Goddard. 2001. Prediction of total genetic value using genome-wide dense marker maps. Genetics 157:1819-1829.

Misztal, I., A. Legarra, and I. Aguilar. 2009. Computing procedures for genetic evaluation including phenotypic, full pedigree, and genomic information. J. Dairy Sci. 92:4648-4655.

Nothnagel, M., D. Ellinghaus, S. Schreiber, M. Krawczak, and A. Franke. 2009. A comprehensive evaluation of SNP genotype imputation. Hum. Genet. 125:163-171.

Ogutu, J., H.-P. Piepho, and T. Schulz-Streeck. 2011. A comparison of random forests, boosting and support vector machines for genomic selection. BMC Proc. 5(Suppl. 3):S11.

Olson, K. M., P. M. VanRaden, M. E. Tooker, and T. A. Cooper. 2011. Differences among methods to validate genomic evaluations for dairy cattle. J. Dairy Sci. 94:2613-2620.

Park, T., and G. Casella. 2008. The Bayesian Lasso. J. Am. Stat. Assoc. 103:681-686.

Pösö, J., and E. A. Mäntysaari. 1996. Relationships between clinical mastitis, somatic cell score, and production for the first three lactations of Finnish Ayrshire. J. Dairy Sci. 79:1284-1291.

Pryce, J. E., and H. D. Daetwyler. 2012. Designing dairy cattle breeding schemes under genomic selection: A review of international research. Anim. Prod. Sci. 52:107-114.

Pryce, J. E., R. F. Veerkamp, R. Thompson, W. G. Hill, and G. Simm. 1997. Genetic aspects of common health disorders and measures of fertility in Holstein Friesian dairy cattle. Anim. Sci. 65:353-360.

Scheet, P., and M. Stephens. 2006. A fast and flexible statistical model for large-scale population genotype data: Applications to inferring missing genotypes and haplotypic phase. Am. J. Hum. Genet. 78:629-644.

Segelke, D., J. Chen, Z. Liu, F. Reinhardt, G. Thaller, and R. Reents. 2012. Reliability of genomic prediction for German Holsteins using imputed genotypes from low-density chips. J. Dairy Sci. 95:5403-5411.

Su, G., R. F. Brøndum, P. Ma, B. Guldbrandtsen, G. P. Aamand, and M. S. Lund. 2012. Comparison of genomic predictions using medium-density $(\sim 54,000)$ and high-density $(\sim 777,000)$ single nucleotide polymorphism marker panels in Nordic Holstein and Red Dairy Cattle populations. J. Dairy Sci. 95:4657-4665.

Sun, C., X.-L. Wu, K. A. Weigel, G. J. M. Rosa, S. Bauck, B. W. Woodward, R. D. Schnabel, J. F. Taylor, and D. Gianola. 2012. An ensemble-based approach to imputation of moderate-density genotypes for genomic selection with application to Angus cattle. Genet. Res. (Camb.) 94:133-150.

VanRaden, P. M. 2008. Efficient methods to compute genomic predictions. J. Dairy Sci. 91:4414-4423.

VanRaden, P. M., D. J. Null, M. Sargolzaei, G. R. Wiggans, M. E. Tooker, J. B. Cole, T. S. Sonstegard, E. E. Connor, M. Winters, J. B. C. H. M. van Kaam, A. Valentini, B. J. Van Doormaal, M. A. Faust, and G. A. Doak. 2013. Genomic imputation and evaluation using high-density Holstein genotypes. J. Dairy Sci. 96:668-678.

VanRaden, P. M., C. P. Van Tassell, G. R. Wiggans, T. S. Sonstegard, R. D. Schnabel, J. F. Taylor, and F. S. Schenkel. 2009. Invited review: Reliability of genomic predictions for North American Holstein bulls. J. Dairy Sci. 92:16-24.

Van Tassell, C. P. V., T. P. L. Smith, L. K. Matukumalli, J. F. Taylor, R. D. Schnabel, C. T. Lawley, C. D. Haudenschild, S. S. Moore, W C. Warren, and T. S. Sonstegard. 2008. SNP discovery and allele frequency estimation by deep sequencing of reduced representation libraries. Nat. Methods 5:247-252.

Vitezica, Z. G., I. Aguilar, I. Misztal, and A. Legarra. 2011. Bias in genomic predictions for populations under selection. Genet. Res. (Camb.) 93:357-366.

Weigel, K. A., G. De los Campos, A. I. Vazquez, G. J. M. Rosa, D Gianola, and C. P. Van Tassell. 2010a. Accuracy of direct genomic values derived from imputed single nucleotide polymorphism genotypes in Jersey cattle. J. Dairy Sci. 93:5423-5435.

Weigel, K. A., C. P. Van Tassell, J. R. O'Connell, P. M. VanRaden, and G. R. Wiggans. 2010b. Prediction of unobserved single nucleotide polymorphism genotypes of Jersey cattle using reference panels and population-based imputation algorithms. J. Dairy Sci. 93:2229-2238.

Wiggans, G. R., P. M. VanRaden, L. R. Bacheller, F. A. Ross Jr., T. S. Sonstegard, G. te Meerman, and C. P. Van Tassell. 2009. Transition of genomic evaluation from a research project to a production system. J. Dairy Sci. 87(E-Suppl. 2):313-314. (Abstr.)

Wiggans, G. R., P. M. VanRaden, and T. A. Cooper. 2011. The genomic evaluation system in the United States: Past, present, future. J. Dairy Sci. 94:3202-3211.

Zhang, Z., and T. Druet. 2010. Marker imputation with low-density marker panels in Dutch Holstein cattle. J. Dairy Sci. 93:54875494. 